\title{
Sensitivity of Initial Thoracentesis for Malignant Pleural Effusion Stratified by Tumor Type in Patients with Strong Evidence of Metastatic Disease
}

\author{
Horiana B Grosu ${ }^{\mathrm{a}}$ Farah Kazzaz $^{\mathrm{b}} \quad$ Erik Vakil $^{\mathrm{a}} \quad$ Sofia Molina ${ }^{\mathrm{a}} \quad$ David Ost $^{\mathrm{a}}$ \\ a Department of Pulmonary Medicine, The University of Texas MD Anderson Cancer Center, Houston, TX, USA; \\ ${ }^{b}$ Department of Internal Medicine, University of Texas Health Science Center at Houston, Houston, TX, USA
}

\section{Keywords}

Pleural effusion · Thoracentesis · Malignant pleural effusion

\begin{abstract}
Background: Thoracentesis with cytological examination of pleural fluid is the initial test of choice for evaluation of pleural effusions in patients with suspected malignant pleural effusion (MPE). There is limited data on the sensitivity of thoracentesis stratified by tumor type. A better understanding of stratified sensitivities is of clinical interest, and may guide early and appropriate referral for pleural biopsy. Objective: The primary objective was sensitivity of thoracentesis with pleural fluid cytology stratified by tumor type. Methods: This is a retrospective cohort study of consecutive patients with a solid tumor malignancy with proven or strong suspicion for metastatic disease with new pleural effusions that underwent an initial thoracentesis. Only patients with metastatic disease were included. Results: Of the 725 patients examined, 63\% had pleural fluid cytology positive for malignancy. Sensitivity of thoracentesis varied from a low of 0.38 (95\% Cl 0.13-0.68) in head and neck malignancy, 0.38 (95\% $\mathrm{Cl} 0.15-0.65)$ in sarcoma, and $0.53(95 \% \mathrm{Cl} 0.34-0.72)$ in renal cancer to a high of $93(95 \% \mathrm{Cl} 88-97)$ in breast cancer, and
\end{abstract}

100 (95\% Cl 0.82-100) in pancreatic cancer. Factors associated with an increased risk of MPE included larger amount of fluid drained ( $p=0.014)$ and higher pleural fluid protein $(p=0.002)$. The only factor associated with decreased risk of MPE if first cytology was negative for malignancy was the presence of contralateral effusion ( $p=0.005)$. Conclusions: Sensitivity of thoracentesis for solid tumors varies significantly depending on the type of tumor and is lowest in those with sarcomas, head and neck malignancies, and renal cell cancers.

(c) 2018 S. Karger AG, Basel

\section{Introduction}

Approximately, 1.5 million new pleural effusions are diagnosed each year in the United States [1]. Thoracentesis with chemical, hematologic, and cytologic analysis of pleural fluid is the most common procedure performed for the diagnosis of pleural effusion not readily explained by clinical history [2]. In patients with cancer, pleural effusion may represent metastatic disease and making a diagnosis is paramount [3]. A definitive diagnosis of malignant pleural effusion (MPE) can be made by thoracentesis

\section{KARGER}

(c) 2018 S. Karger AG, Basel

E-Mail karger@karger.com

www.karger.com/res
Horiana B. Grosu, MD

Department of Pulmonary Medicine, Unit 1462

The University of Texas MD Anderson Cancer Center

1515 Holcombe Blvd, Houston, TX 77030 (USA)

E-Mail hbgrosu@mdanderson.org 
with analysis of pleural fluid cytology or histologic analysis of pleural biopsies.

The diagnostic yield for malignancy by analysis of pleural fluid cytology obtained on initial thoracentesis is $40-60 \%$, and the yield increases only marginally for subsequent thoracenteses [4-6]. Sensitivity of pleural fluid cytology obtained on initial thoracentesis is 60\% [5]. Pleural biopsy, either image-guided percutaneous or surgical, is recommended in cases where analysis of pleural fluid cytology is negative and clinical suspicion for MPE remains high [7]. Image-guided percutaneous pleural biopsy of focal pleural thickening and/or nodules has a reported sensitivity for malignancy of $70-94 \%$ and specificity approaching $100 \%$ [8-11]. Thoracoscopy with pleural biopsy under direct visualization is considered the gold standard for the diagnosis of MPE, with reported sensitivity from 93 to $100 \%[12,13]$.

Currently, thoracentesis is the recommended test for MPE due to all solid tumors. However, while thoracoscopy sensitivity is consistently high for all solid tumors, we hypothesized that the sensitivity of thoracentesis varies between types of solid tumors. This is clinically relevant, since if the marginal gain in sensitivity is significant, then a more aggressive strategy of early thoracoscopy would be justified.

Despite its modest sensitivity compared with thoracoscopic pleural biopsy, thoracentesis is considered the initial test of choice given its relative simplicity, safety, and cost. However, while thoracoscopy sensitivity is consistently high for all solid tumors [12], we hypothesized that the sensitivity of thoracentesis varies between types of solid tumors. This is clinically relevant, since if the marginal gain in sensitivity is significant, then a more aggressive strategy of early thoracoscopy would be justified. If we could identify cases in which thoracentesis has particularly low sensitivity, direct referral for thoracoscopic biopsy would be a medically appropriate and cost effective alternative strategy. This has indeed proven to be the case for mesothelioma [14]. The sensitivity of thoracentesis in patients with mesothelioma is between 16 and $30.8 \%$, since it is difficult to differentiate between reactive mesothelial cells secondary to inflammation and malignant cells $[5,15]$. The diagnostic sensitivity of thoracentesis may also differ between lung cancer sub-types, with metastatic adenocarcinoma having a greater sensitivity than squamous cell carcinoma $[5,7]$.

The aim of our study is to evaluate the sensitivity of initial thoracentesis with analysis of pleural fluid cytology stratified by tumor type in patients with an existing diagnosis of cancer and high clinical suspicion of MPE.

\section{Materials and Methods}

This retrospective cohort study used data of consecutive patients with pleural effusions who underwent an initial thoracentesis for suspected MPE from January 1, 2010, to December 31, 2013 at our institution. Our Institutional Review Board (Committee 4 PA17-0384) approved this study.

We included all patients 18 years of age or older who had a known diagnosis of cancer and had either proven metastatic disease or strong clinical evidence of metastatic disease. Strong clinical evidence of metastatic disease was defined as imaging demonstrating multiple metastases in a typical clinical pattern, such as a positron emission tomography (PET) or bone scan showing multiple bone metastases, computed tomography scan (CT) or magnetic resonance image of the brain showing metastatic disease, and computed tomography scan of the chest/abdomen or pelvis with findings sufficiently definitive that the patient was deemed to have metastatic disease. In order to maximize the ability to detect a meaningful difference in the sensitivity of initial thoracentesis to diagnose MPE stratified by tumor type, we elected to enrich our cohort with patients who had either proven metastatic cancer or strong clinical evidence of metastatic disease and had a strong clinical suspicion for MPE.

We excluded patients lost to follow-up; patients without a chest $\mathrm{x}$-ray, CT scan, or PET scan within 14 days from initial thoracentesis; patients with no history or suspicion of cancer; patients with loculated pleural effusions on chest x-ray as we would not quantify with precision the size of effusion in these patients; patients with a previous thoracentesis at an outside institution or history of chest tube placement; and patients with hematologic malignancy.

Pleural effusion size was based on the most recent chest x-ray done within 2 weeks of the first thoracentesis and was categorized as follows:

- Blunting of costophrenic angle, but at least part of diaphragm is still visible.

- Effusion greater than 1, and up to the inferior border of the vascular pedicle.

- Effusion greater than 2, and up to the top of the cardiac silhouette.

- Effusion greater than 3, and up to the top of the aortic arch.

- Highest effusion greater than aortic arch.

Pleural fluid cytology was processed using both smears and cell block was performed. For details of this procession see online supplementary appendix S1 (for all online suppl. material, see www. karger.com/doi/10.1159/000490732).

The primary outcome was the sensitivity of initial thoracentesis by tumor type. For our analysis, all initial cytology results positive for malignancy were considered true positives.

The final designated etiology of pleural effusion in all patients was established by the treating physician and pulmonary research team based on review of all available clinical information and with follow-up for at least 6 months. The final diagnostic categories were as follows:

\section{Malignant Pleural Effusion}

Malignant cells on initial pleural fluid cytology or during a subsequent thoracentesis or on histopathologic assessment of pleural biopsies performed within 6 months from the initial thoracentesis. The initial results was considered a false negative result if malignancy was diagnosed on subsequent work up within 6 months from the first thoracentesis. 
Specific Benign Effusion

Pleural effusions with characteristics consistent with chemotherapy-induced pleuritis, radiation-induced pleuritis, paramalignant pleural effusions, or infectious pleuritis. Definitions for specific benign effusion were previously described in cases where a pleural biopsy was done [16]. In those cases where pleural biopsy was not done, the final diagnosis was assigned by the multidisciplinary oncology and pulmonary team on the basis of a review of their clinical, pathologic, microbiologic, and radiologic data, with follow-up for at least 6 months. The final diagnostic categories were as follows:

- Chemotherapy-induced pleuritis: Pleural effusion that develops in patients receiving treatment with chemotherapeutic agents associated with pleural effusions, who did not also receive radiation to the clinically relevant pleural region, had no other cause for pleural effusion and in whom pleural effusion resolved with cessation of the drug. Chemotherapeutic agents associated with pleural effusions were identified using published Tables $[17,18]$ and drugs not appearing in these tables were cross-checked using an online database [19].

- Radiation-induced pleuritis: Pleural effusion that develops in a patient who received prior radiation therapy within 18 months of radiation treatment, whose effusion exclusively involved the lung and pleura that were irradiated, did not also receive chemotherapy known to cause pleural effusion, and had no other cause for pleural effusion.

- Paramalignant pleural effusion: Pleural effusion that develops in a patient who received prior therapy for their malignancy but did not meet criteria for chemotherapy or radiation-induced pleuritis. This includes patients who received both chemotherapy and radiation or who could not be placed in one category only. Patients with end organ damage due to treatment effects such as volume overload and when pleural effusion develop in association with atelectasis, pulmonary embolism, or hepatic hydrothorax where malignancy was felt to be the primary cause.

- Infectious pleuritis: Pleural effusion secondary to an infectious cause determined directly by pleural fluid cultures or histopathology or tissue culture.

\section{Unknown Etiology}

Pleural effusion where a cause or presumed cause of effusion was not found and patients did not show evidence of MPE during follow-up. These effusions were considered benign at the end of the follow-up period, which concluded with resolution of the effusion or after a minimum of 6 months.

\section{Statistics}

Descriptive statistics were used to summarize the demographic and clinical characteristics of all patients in the study. Normally distributed continuous variables are expressed as the mean \pm SD. Medians were used for non-normally distributed data. Frequencies were used for categorical data, which were compared using the Fisher exact test. The sensitivity, specificity, positive predictive value, and negative predictive value were estimated for all patients. Univariate and multivariate logistic regressions were used to evaluate factors associated with a final diagnosis of malignancy and with negative first cytology. Variables with a p-value of less than 0.2 on univariate analysis were considered candidate variables for the multivariate regression model. Backward selection, with a
Table 1. Cytology yield by tumor type in patients with solid tumor malignancy and/or strong clinical suspicion of malignant pleural effusion

\begin{tabular}{lcc}
\hline Type of cancer & $\begin{array}{c}\text { Cytology positive } \\
\text { for malignancy, } \%\end{array}$ & $95 \%$ CI \\
\hline Mesothelioma & $0 / 5(0)$ & $0.005-0.716$ \\
Sarcoma & $6 / 29(20)$ & $0.079-0.397$ \\
Head and neck & $5 / 23(21)$ & $0.074-0.437$ \\
Renal & $16 / 43(37)$ & $0.229-0.532$ \\
Lung, squamous cell & $9 / 23(39)$ & $0.197-0.614$ \\
Other solid & $7 / 16(43)$ & $0.197-0.701$ \\
Prostate & $6 / 13(46)$ & $0.192-0.748$ \\
Melanoma & $10 / 21(47)$ & $0.257-0.702$ \\
Multiple solid & $38 / 74(51)$ & $0.394-0.631$ \\
Lung, small cell & $7 / 13(54)$ & $0.251-0.807$ \\
Unknown primary & $6 / 11(54)$ & $0.233-0.832$ \\
Colorectal & $20 / 37(55)$ & $0.369-0.705$ \\
Esophageal & $13 / 23(56)$ & $0.344-0.768$ \\
Lung, large cell & $14 / 24(58)$ & $0.366-0.778$ \\
Cervical, squamous cell & $4 / 6(66)$ & $0.222-0.956$ \\
Hepatic & $4 / 6(66)$ & $0.222-0.956$ \\
Lung unspecified & $5 / 7(71)$ & $0.290-0.963$ \\
Gastric & $11 / 15(73)$ & $0.448-0.922$ \\
Endometrial & $6 / 8(75)$ & $0.349-0.968$ \\
Thyroid & $10 / 13(77)$ & $0.461-0.949$ \\
Lung, adenocarcinoma & $71 / 91(78)$ & $0.681-0.860$ \\
Urothelial carcinoma & $5 / 6(83)$ & $0.358-0.995$ \\
Ovarian & $26 / 31(84)$ & $0.662-0.945$ \\
Breast & $140 / 165(85)$ & $0.784-0.899$ \\
Pancreatic & $19 / 22(86)$ & $0.650-0.970$ \\
\hline & & \\
& &
\end{tabular}

$p$ value of $<0.05$ to stay in the model, was used to arrive at a parsimonious multivariable model.

$p$ values less than. 05 were considered significant. All tests were 2-sided.

\section{Results}

We reviewed the medical records of 1,323 patients with cancer that was either proven to be metastatic or in whom strong clinical evidence of metastatic disease was present. We excluded 107 patients with congestive heart failure and 373 with high clinical suspicion of pneumonia. Of the remaining 843 patients, 725 had solid tumor malignancies and were included in our final analysis.

Of our final cohort, 458 (63\%) had an initial thoracentesis with pleural fluid cytology positive for malignancy. Diagnostic yield of pleural fluid cytology was greatest for pancreatic cancer (86 [95\% CI 65-97]) and lowest for sarcoma (20 [95\% CI 5-71]; Table 1). After final review of all available clinical information with follow-up for at least 
Table 2. Sensitivity and specificity by tumor type in patients with solid tumor malignancy and/or strong clinical suspicion of malignant pleural effusion

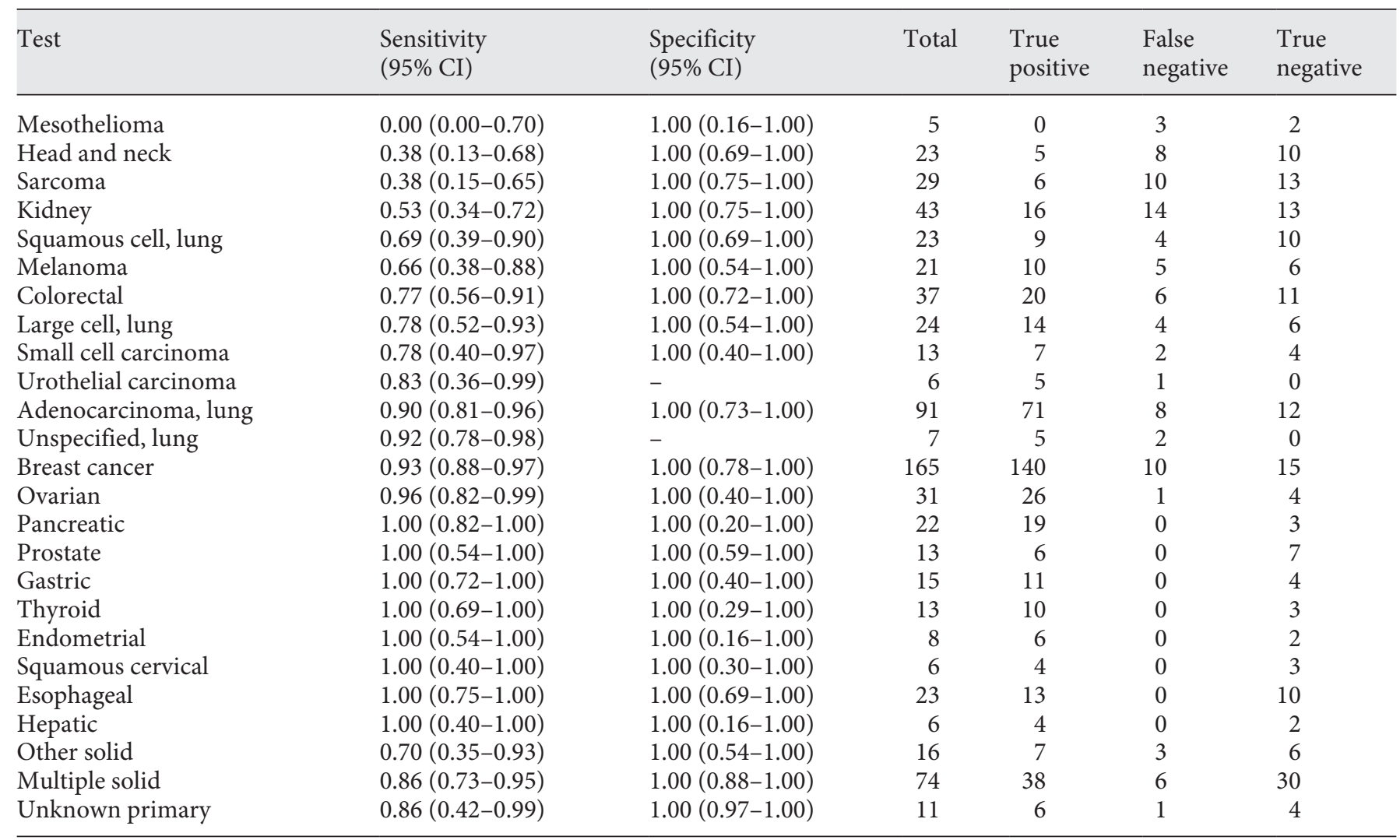

All positive cytologies were considered true positives.

6 months, a final diagnosis of malignancy was established in 537 of 725 (74\%) patients. The median time of followup for effusions with unknown etiology was 247 days (range from 15 to 915 days).

Performance characteristics of pleural fluid cytology were calculated for each tumor type (Table 2). The sensitivity of thoracentesis was lowest for head and neck malignancies (0.38 [95\% CI 0.13-0.68]), sarcoma (0.38 [95\% CI $0.15-0.65])$, and renal cancer $(0.53$ [95\% CI $0.34-$ $0.72]$ ). The sensitivity for mesothelioma was 0 ; however, our cohort included only 5 cases.

We evaluated the risk factors of the entire cohort for final diagnosis of MPE as final diagnosis. On univariate analysis, the volume of pleural fluid drained $(p=0.001)$, pleural fluid lactate dehydrogenase ( $\mathrm{LDH} ; p=0.023)$, pleural protein level $(p<0.0001)$, and pleural cholesterol level $(p=<0.0001)$ significantly impacted the odds of having a final diagnosis of MPE. On multivariate analysis, amount of fluid drained and higher protein level remained as risk factors of having a final diagnosis of MPE (Table 3).
In addition, we evaluated risk factors of having a final diagnosis of MPE in those patients with a negative first cytology (Table 4).

\section{Discussion}

The diagnostic sensitivity of initial thoracentesis with pleural fluid cytology in patients with cancer and strong clinical suspicion of MPE depends on tumor type. Sensitivity is low for patients with sarcoma, head and neck malignancies, kidney cancer, and mesothelioma; and high in patients with breast and pancreatic cancer.

Our findings are corroborated by a retrospective study by Bielsa et al. [5] who also reported different sensitivities of thoracentesis stratified by tumor type, with the highest sensitivity for adenocarcinoma (66.5\%). However, the sensitivity for adenocarcinomas of all organ types in our cohort was considerably greater than $66.5 \%$ [5]. An explanation for higher sensitivity may be the use of cell blocks 
Table 3. Risk Factors for malignant pleural effusion as final diagnosis in patent with pleural effusion

\begin{tabular}{|c|c|c|c|c|c|c|c|c|}
\hline Covariate & \multicolumn{4}{|c|}{ Univariate logistic regression } & \multicolumn{4}{|c|}{ Multivariate logistic regression } \\
\hline Radiation within 30 days prior to tap & 1.23 & 0.730 & 2.099 & 0.427 & & & & \\
\hline Chemotherapy within 30 day after tap & 1.53 & 1.097 & 2.146 & 0.012 & & & & \\
\hline Radiation within 30 days after tap & 1.05 & 0.618 & 1.805 & 0.840 & & & & \\
\hline \multicolumn{9}{|l|}{ Size of the effusion on chest X-ray* } \\
\hline Blunting of costophrenic angle & 1.00 & & & & & & & \\
\hline Vascular pedicle & 1.43 & 0.695 & 2.969 & 0.327 & & & & \\
\hline Cardiac silhouette & 2.00 & 0.980 & 4.088 & 0.057 & & & & \\
\hline Aortic arch & 3.24 & 1.402 & 7.496 & 0.006 & & & & \\
\hline Highest & 2.27 & 0.898 & 5.749 & 0.083 & & & & \\
\hline Amount of fluid drained, $\mathrm{mL}$ & 1.00 & 1.000 & 1.001 & $<0.001$ & 1.00 & 1.000 & 1.000 & 0.014 \\
\hline
\end{tabular}

Table 4. Risk Factors for malignant pleural effusion as final diagnosis in patent with negative first cytology

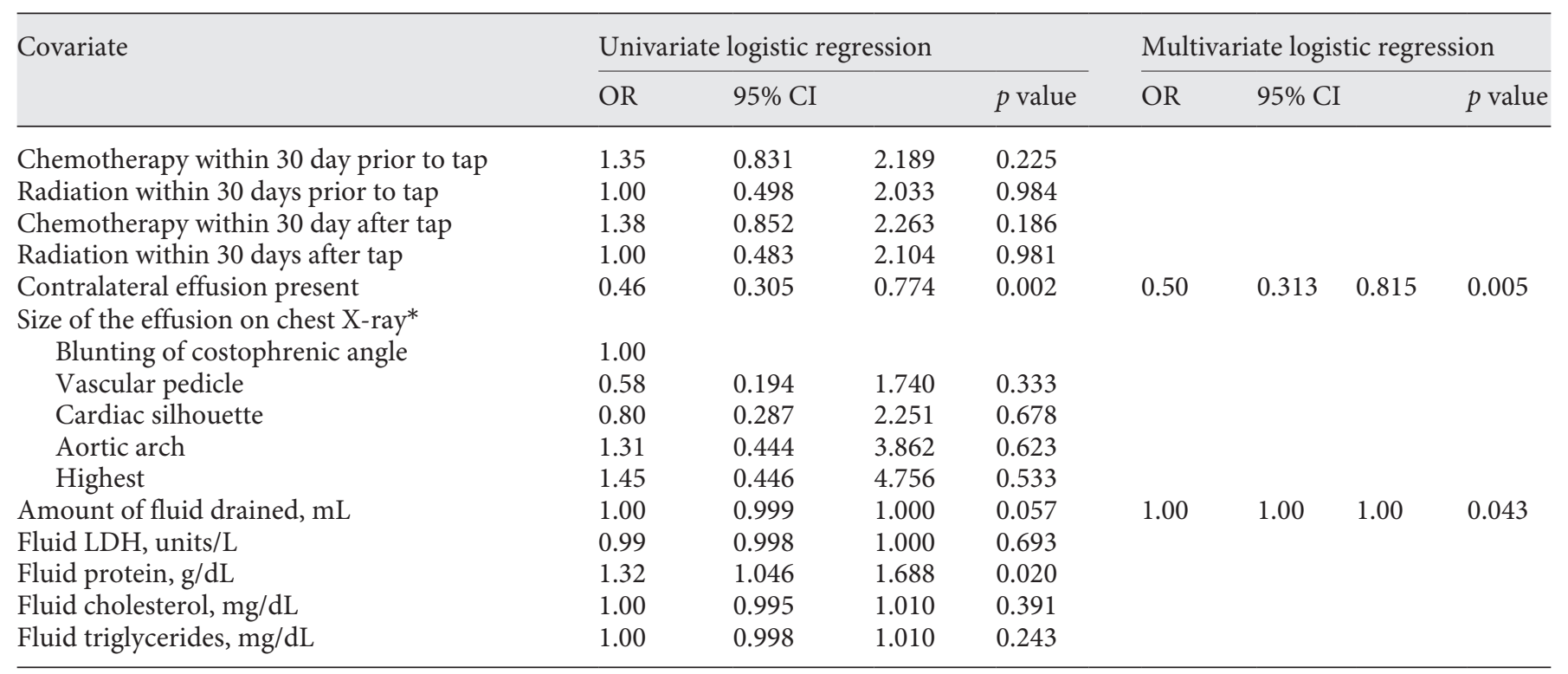

in addition to smears rather than only smears for pleural fluid cytological analysis. By current standards, cell blocks are essential for the comprehensive evaluation of pleural fluid cytology since they are made from concentrated cellular samples and may be used for immunocytochemistry and molecular testing [20,21]. All pleural fluid cytology evaluations in our cohort included cell block analysis in addition to smears as compared with those by Bielsa et al.
[5] that relied only on conventional smears. The superiority of using both smears and cell block was also proposed by Porcel et al. [22].

In our study, as in most studies that report on the sensitivity of thoracentesis for MPE, the use of all available clinical information and follow-up of at least 6 months was used as the reference standard of truth for determining what constituted a true negative result $[6,23-25]$. We 
chose 6 months rather than a longer follow-up due to the nature of our patient population with already evidence of metastatic disease and low likelihood of survival beyond 6 months. The sensitivity of pleural fluid analysis in prior studies has ranged from 40 to $80 \%$ [8-20]. To our knowledge, only one study, published in abstract form, has systematically reported on the sensitivity of pleural fluid cytology using pleural biopsy on thoracoscopy as the reference standard [19]. They report a sensitivity of 0.70 ( $95 \%$ CI 0.59-0.80); however, information on previous thoracenteses and other relevant clinical parameters were not available, limiting the interpretation of these results.

The sensitivity of a test when applied to a population is the weighted average of the sensitivity of the test for the different strata. Therefore, it is important to present strataspecific estimates of the measures of disease accuracy for relevant subgroups $[26,27]$. In the case of thoracentesis for MPE, if sarcoma or mesothelioma is very rare or non-existent in the population studied, then the weighted average will be higher since thoracentesis has low sensitivity for MPE in these diseases. For instance, in the study by Benamore et al. [9], the authors report very low sensitivity for cytological examination for malignancy but their prevalence of mesothelioma was high, while in the study by Salyer et al. [24] the sensitivity for cytological examination for malignancy was high but the prevalence of mesothelioma was low and that of adenocarcinoma was high. This likely explains the heterogeneity of results in prior studies.

Our study suggests there are clinically meaningful differences in the sensitivity of thoracentesis between different types of tumors. Therefore, the utility of a diagnostic thoracentesis when malignancy is suspected may change depending on the underlying malignancy. For example, in a patient with sarcoma, where the sensitivity of thoracentesis is low for MPE, direct referral for pleural biopsy may be appropriate.

Similar to other studies we found that larger amount of fluid drained, higher pleural fluid protein and higher pleural fluid LDH were associated with a higher risk of MPE [28]. LDH is found in numerous clinical conditions and had been reported as a marker of poor outcome in cancer patients [29]. The proposed explanation for its rise in cancer is the favored use of glycolysis for energy, instead of oxidative phosphorylation by tumor cells, which is mediated by LDH [30]. However, this is not true from those patients in whom the first cytology was negative and final diagnosis was MPE. In this selected group of people with negative cytology, having contralateral pleural effusion was found to decrease the risk of having a final diagnosis of MPE.
Most clinicians would be interested in knowing the sensitivity of thoracentesis in those patients with no evidence of metastatic disease; however, we made the decision to include only patients with already evidence of metastatic disease as these are the patients with a high pretest probability of MPE. This is however a limitation of our study as it includes the narrow generalizability of our results to other settings. We speculate that in a different population, where the pretest probability of MPE is low, the yield of pleural fluid cytology on initial thoracentesis stratified by tumor type is expected to be lower than what we have found in this study. Another limitation is that we relied on evaluation of all available clinical information and patient follow-up as the reference standard of truth for a true negative result rather than on pleural biopsy, particularly surgical biopsy as not all patients had a pleural biopsy. It is therefore possible that some effusions recorded as true negatives were actually false negatives. This would decrease the sensitivity of thoracentesis further for those tumors with already low sensitivities. Another limitation was the uncertainty of the estimate as in some cases the CI are very wide and this is this is due to the low number of patient in each diagnostic group. The generalizability of our results may also be impacted by the resources, expertise, and specimen handling methods available at different institutions as our study population received care at a dedicated cancer center.

In conclusion, in patients with a high pre-test probability of MPE secondary to a solid tumor malignancy, the sensitivity of pleural fluid cytology on initial thoracentesis to diagnosis of MPE is significantly impacted by the underlying cancer type. Referring patients with MPE with tumors associated with very low thoracentesis sensitivity such as those with sarcoma, head and neck malignancies, kidney cancer, mesothelioma, and squamous cell carcinoma directly to a thoracoscopic or image-guided pleural biopsy may be considered in appropriate clinical settings. Future work such as cell-free DNA might improve our knowledge in this area of clinical practice and increase the sensitivity of the diagnosis in patients with suspected MPE.

\section{Authors Contributions}

D.O. and H.B.G. were principal investigator and was responsible for study design, analysis, management, writing, and editing. F.K., E.V., and S.M. were responsible for data collection, study oversight, analysis, and writing. All authors participated in reviewing and editing the manuscript. 


\section{References}

1 Light RW: Clinical practice. Pleural effusion. N Engl J Med 2002;346:1971-1977.

2 Owings MF, Kozak LJ: Ambulatory and Inpatient Procedures in the United States, 1996. Vital and Health Statistics Series 13, Data from the National Health Survey, 1998, pp 1-119.

3 Kvale PA, Selecky PA, Prakash UB: Palliative care in lung cancer: ACCP evidence-based clinical practice guidelines (2nd edition). Chest 2007;132:368S-403S.

4 Clive AO, Kahan BC, Hooper CE, Bhatnagar R, Morley AJ, Zahan-Evans N, Bintcliffe OJ, Boshuizen RC, Fysh ET, Tobin CL, Medford AR, Harvey JE, van den Heuvel MM, Lee YC, Maskell NA: Predicting survival in malignant pleural effusion: development and validation of the LENT prognostic score. Thorax 2014; 69:1098-1104.

5 Bielsa S, Panades MJ, Egido R, Rue M, Salud A, Matias-Guiu X, Rodriguez-Panadero F, Porcel JM: (Accuracy of pleural fluid cytology in malignant effusions). An Med Interna 2008; $25: 173-177$.

6 Prakash UB, Reiman HM: Comparison of needle biopsy with cytologic analysis for the evaluation of pleural effusion: analysis of 414 cases. Mayo Clin Proc 1985;60:158-164.

7 Hooper C, Lee YC, Maskell N: Investigation of a unilateral pleural effusion in adults: British Thoracic Society Pleural Disease Guideline 2010. Thorax 2010;65(suppl 2):ii4-ii17.

8 Adams RF, Gray W, Davies RJ, Gleeson FV: Percutaneous image-guided cutting needle biopsy of the pleura in the diagnosis of malignant mesothelioma. Chest 2001;120:17981802.

9 Benamore RE, Scott K, Richards CJ, Entwisle JJ: Image-guided pleural biopsy: diagnostic yield and complications. Clin Radiol 2006;61: 700-705.

10 Scott EM, Marshall TJ, Flower CD, Stewart S Diffuse pleural thickening: percutaneous CTguided cutting needle biopsy. Radiology 1995; 194:867-870.
11 Mueller PR, Saini S, Simeone JF, Silverman SG, Morris E, Hahn PF, Forman BH, McLoud TC, Shepard JO, Ferrucci JT Jr: Image-guided pleural biopsies: indications, technique, and results in 23 patients. Radiology 1988;169:14.

12 Harris RJ, Kavuru MS, Rice TW, Kirby TJ: The diagnostic and therapeutic utility of thoracoscopy. A review. Chest 1995;108:828841.

13 Rahman NM, Ali NJ, Brown G, Chapman SJ, Davies RJ, Downer NJ, Gleeson FV, Howes TQ, Treasure T, Singh S, Phillips GD: Local anaesthetic thoracoscopy: British Thoracic Society Pleural Disease Guideline 2010. Tho$\operatorname{rax}$ 2010;65(suppl 2):ii54-ii60.

14 Husain AN, Colby TV, Ordonez NG, Allen TC, Attanoos RL, Beasley MB, Butnor KJ, Chirieac LR, Churg AM, Dacic S, GalateauSalle F, Gibbs A, Gown AM, Krausz T, Litzky LA, Marchevsky A, Nicholson AG, Roggli VL, Sharma AK, Travis WD, Walts AE, Wick MR: Guidelines for pathologic diagnosis of malignant mesothelioma 2017 update of the consensus statement from the international mesothelioma interest group. Arch Pathol Lab Med 2018;142:89-108.

15 Walters J, Maskell NA: Biopsy Techniques for the Diagnosis of Mesothelioma; in Tannapfel A (ed): Malignant Mesothelioma. Springer Berlin Heidelberg, Berlin, Heidelberg, 2011, pp 45-55.

16 Vakil E, Ost D, Vial MR, Stewart J, Sarkiss MG, Morice RC, Casal RF, Eapen GA, Grosu HB: Non-specific pleuritis in patients with active malignancy. Respirology 2018;23:213219.

17 Light R: Pleural Diseases (ed 6). Philadelphia, Lippincott Williams \& Wilkins, 2013.

18 Moschos C, Kalomenidis I: Drug related pleural disease. Pleural Bulletin, Turk Toraks Denergi, 4.

19 Camus P, Foucher P: Pneumotox: The drug induced respiratory disease website, 2012-v2, 2017. http://www.pneumotox.com/.
20 Jain D, Mathur SR, Iyer VK: Cell blocks in cytopathology: a review of preparative methods, utility in diagnosis and role in ancillary studies. Cytopathology 2014;25:356-371.

21 Shivakumarswamy U, Arakeri SU, Karigowdar MH, Yelikar B: Diagnostic utility of the cell block method versus the conventional smear study in pleural fluid cytology. J Cytol 2012;29:11-15.

22 Porcel JM, Quiros M, Gatius S, Bielsa S: Examination of cytological smears and cell blocks of pleural fluid: complementary diagnostic value for malignant effusions. Rev Clin Esp 2017;217:144-148.

23 Hirsch A, Ruffie P, Nebut M, Bignon J, Chretien J: Pleural effusion: laboratory tests in 300 cases. Thorax 1979;34:106-112.

24 Salyer WR, Eggleston JC, Erozan YS: Efficacy of pleural needle biopsy and pleural fluid cytopathology in the diagnosis of malignant neoplasm involving the pleura. Chest 1975 ; 67:536-539.

25 Nance KV, Shermer RW, Askin FB: Diagnostic efficacy of pleural biopsy as compared with that of pleural fluid examination. Mod Pathol 1991;4:320-324.

26 Coughlin SS, Trock B, Criqui MH, Pickle LW, Browner D, Tefft MC: The logistic modeling of sensitivity, specificity, and predictive value of a diagnostic test. J Clin Epidemiol 1992;45:1-7.

27 Diamond GA: Clinical epistemology of sensitivity and specificity. J Clin Epidemiol 1992; 45:9-13.

28 Verma A, Abisheganaden J, Light RW: Identifying malignant pleural effusion by a cancer ratio (serum LDH: pleural fluid ADA ratio). Lung 2016;194:147-153.

29 Johnson PW, Joel SP, Love S, Butcher M, Pandian MR, Squires L, Wrigley PF, Slevin ML: Tumour markers for prediction of survival and monitoring of remission in small cell lung cancer. Br J Cancer 1993;67:760-766.

30 Gatenby RA, Gillies RJ: Why do cancers have high aerobic glycolysis? Nat Rev Cancer 2004; 4:891-899. 